\title{
Article
}

\section{«Mauvaises herbes », qualité de l'eau et entretien des espaces}

\author{
Marie-Jo Menozzi \\ Docteur en anthropologie, consultante, 12 rue Jules Soufflet, 35310 Rennes, France
}

Élargir les connaissances sur les villes par l'interdisciplinarité est un des enjeux scientifiques de NSS. En prenant les «mauvaises herbes » pour objet de recherche, ce texte d'ethnologue nous permet de prolonger le dossier ouvert dans le dernier numéro de 2006 de la revue $(N S S, 14,4)$ sur la gestion durable des milieux urbains. À travers cette question, modeste en apparence : gérer la végétation spontanée, on comprend que le problème posé par l'usage des pesticides concerne aussi la ville et qu'une gestion responsable de l'environnement urbain doit aller contre la nature belle et propre que préfèrent les citadins.

La Rédaction

\section{Mots-clés :}

plantes; ethnobotanique ; espaces; socio-technique; milieu urbain

\section{Keywords:}

Weeds; ethnobotany; urban spaces; socio-technical aspects
Résumé - Les plantes spontanées sont souvent jugées indésirables en ville et éliminées, du fait qu'elles ne répondent pas aux critères attendus dans les espaces urbains : l'ordre et la propreté. La volonté de réduire les pollutions par les produits chimiques conduit à mettre en place d'autres pratiques : soit laisser pousser, ce qui heurte les représentations sociales dominantes de l'espace urbain; soit utiliser d'autres techniques que les herbicides. La réticence vis-à-vis de l'abandon de ces produits illustre les représentations dont ces techniques sont l'objet, perçues comme une manifestation de progrès. Les «mauvaises herbes » font aussi partie des relations sociopolitiques entretenues au sein de la ville. Ainsi, les modalités techniques d'entretien mises en place sont-elles retraduites par les acteurs dans un registre sociopolitique.

\begin{abstract}
Weeds, water quality and urban space management. Our paper studies the interconnections between phenomena that have seldom been considered simultaneously. Taking the example of weeds in a situation of changing maintenance methods in urban spaces, we show that technical choices are not only driven by technical but also by social and cultural considerations. Mental representations of urban spaces, categorization of plants, representations of techniques as well as political relations in the city are all closely interlinked. Changes in the maintenance and upkeep methods of urban spaces are dictated by the ecological context as well as by the social and cultural one. Changing techniques such as herbicide application therefore implies that the overall context is considered and not solely the technical factors. Spontaneous plants are often deemed undesirable in cities and eliminated because they do not conform to urban criteria of order and cleanliness. To avoid using chemicals, alternative practices can be applied: let the weeds be or use other eradication methods. However, professionals do not easily give up pesticides owing to cultural patterns such as the rule that a good worker does not leave weeds around or to beliefs about pesticides as a hallmark of progress. Weeds are also involved in the socio-political relationships of urban space. The technical methods used are socially interpreted: for instance letting weeds grow in a given area while they are eradicated in another is interpreted as a lack of interest by the municipality for people living in areas where weeds are not eliminated.
\end{abstract}

Auteur correspondant : marie-jo.menozzi@wanadoo.fr 


\section{Introduction}

Les publicités relatives aux produits désherbants nous offrent des images de paradis faits de pelouses vertes dénuées de toute "mauvaise herbe». Ces produits rendent les fleurs resplendissantes, les chiens heureux et les propriétaires épanouis. Revers de la médaille, fait oublié de ces publicités, une partie de ces produits utilisés se retrouvent dans les cours d'eau par un phénomène de ruissellement et contribuent à leur pollution.

Les communes de Bretagne, région qui souffre d'un problème de qualité de l'eau, se trouvent de plus en plus confrontées à une contradiction. Comme la plupart des villes actuellement, elles doivent répondre à une demande esthétique spécifique - des fleurs, des massifs, des parterres, des haies, des espaces verts -, qui engage certaines normes, notamment celles relatives à l'ordre et à la propreté. Pour cela, la solution est d'utiliser des produits phytosanitaires, en particulier des produits désherbants, afin d'éliminer les "mauvaises herbes " qui nuisent à la qualité attendue des espaces. Mais ces pratiques urbaines, du fait de la présence importante de surfaces imperméabilisées, contribuent pour une part non négligeable à la pollution des eaux par des pesticides ${ }^{1}$. On retrouve dans les cours d'eau en aval des espaces urbains des taux élevés de molécules comme le glyphosate, couramment utilisé dans l'entretien des jardins, de la voierie et des espaces verts pour le désherbage. Des expérimentations conduites dans des communes de l'agglomération rennaise montrent une grande sensibilité des zones urbaines, comme les routes, les rues, les trottoirs, les aires gravillonnées... au ruissellement et à la pollution par les pesticides, notamment le glyphosate et le diuron. L'abandon d'un certain nombre de molécules, perçues comme les plus dangereuses, s'est traduit par une augmentation de l'utilisation du glyphosate en substitution des produits supprimés (180\% d'augmentation entre 1996 et 1999). Les impacts des désherbages urbains, par les communes ou les particuliers, se traduisent par une augmentation des teneurs des molécules qu'on retrouve dans les cours d'eau en aval des communes (Observatoire de l'eau, 2004). Les normes de concentration de molécules de pesticides sont de $0,1 \mu / 1$ pour chaque molécule et de $0,5 \mu / 1$ toutes molécules confondues. Les analyses

\footnotetext{
${ }^{1}$ Le terme pesticide « est devenu au $\mathrm{XX}$ e siècle le terme générique utilisé pour désigner toutes les substances naturelles ou de synthèse capables de contrôler, d'attirer, de repousser, de détruire ou de s'opposer au développement des organismes vivants (microbes, animaux ou végétaux) considérés comme indésirables pour l'agriculture, l'entomologie urbaine (par exemple, les cafards dans les habitations), la santé publique (les insectes sont vecteurs de maladies tel le paludisme) ou les surfaces non agricoles (routes, aéroports, voies ferrées...) » (http://www.techno-science.net/ ?onglet=glossaire\&definition =3528). Parmi les pesticides, on peut distinguer les fongicides, les herbicides, les insecticides...
}

réalisées dans les cours d'eau en amont et en aval de Rennes montrent des concentrations la plupart du temps supérieures aux normes $(3,61 \mu / 1$ en juin $1998 ; 0,76 \mu / 1$ en juin $2001 ; 1,92 \mu / 1$ en mars 2003 dans la Vilaine en aval de Rennes). Entre 1998 et 2004, sur quatre prélèvements effectués en aval de la ville, deux sont en deçà de la norme, les autres varient entre 0,15 et $0,4 \mu / 1$ pour le glyphosate ${ }^{2}$.

Comment concilier ces deux aspects apparemment inconciliables, changer les techniques d'entretien tout en conservant aux espaces un aspect acceptable pour les habitants : changer les techniques d'entretien ou modifier la perception des espaces urbains? Ce questionnement, non strictement technique, a été le point de départ d'une étude sociologique sur la perception des «mauvaises herbes » en ville, dans le cadre d'un projet d'entretien des espaces sans produit phytosanitaire ${ }^{3}$.

\section{Présentation du contexte et méthodologie}

Depuis plus de dix ans, dans un souci de préservation de la qualité de l'eau et de la biodiversité, la ville de Rennes s'est engagée dans une démarche de gestion différenciée des espaces (Encadré). Afin d'aller plus loin, dans le cadre de la charte de l'environnement de la ville et du programme Bretagne Eau pure ${ }^{4}$, le projet d'« adaptation de l'entretien des espaces publics aux exigences de qualité des eaux », aussi intitulé « zéro phyto », a été mis en place dans deux quartiers de la ville. L'objectif de ce projet pilote est de ne plus du tout utiliser de produits phytosanitaires, qu'il s'agisse des espaces publics ou des espaces privés, dans un souci de restauration de la qualité de l'eau, et notamment d'abandonner l'usage de désherbant pour éliminer les "mauvaises herbes " présentes en ville. Pour atteindre cet objectif, deux démarches ont été mises en place, l'une portant sur un changement de techniques, l'autre consistant en une réflexion sur les moyens de modifier le regard porté sur les plantes spontanées,

\footnotetext{
${ }^{2}$ Données fournies par J. Especel, service hygiène et santé, ville de Rennes.

${ }^{3}$ Cet article résulte d'un travail réalisé pour le compte de la ville de Rennes, dans le cadre de la mise en place d'actions de sensibilisation aux effets des produits pesticides sur la qualité de l'eau, préalables à la réduction de leur usage. La ville avait exprimé le souhait de mieux connaître les freins et les leviers aux changements de pratiques et de perception des espaces. Pour cela, elle s'est orientée vers la réalisation d'un diagnostic sociologique. Ce travail a fait l'objet d'un rapport intitulé : Adaptation de l'entretien des espaces publics aux exigences de qualité des eaux : diagnostic social et mise en place d'une démarche participative (Idea Recherche, Ceresa, 2002).

${ }^{4}$ Le programme Bretagne Eau pure a été initié par divers partenaires institutionnels (région, conseil généraux, chambres d'agriculture, agence de l'eau Loire-Bretagne, communes...) dans un objectif de "reconquête de la qualité des eaux en Bretagne».
} 


\section{Encadré. La gestion différenciée des espaces}

La méthode de la gestion différenciée a été mise au point afin de favoriser une gestion plus écologique des espaces verts de la ville, et dans un objectif d'entretien plus économique de ces derniers. D'abord mise en place dans des pays du Nord de l'Europe, elle a été adoptée dans quelques villes de France. Rennes fait partie de celles qui ont fait ce choix à partir des années 1980.

Dans une politique de gestion différenciée, tous les espaces ne sont pas traités de la même manière, mais en fonction de leur localisation et de leur usage. Un «code qualité » est élaboré, allant de zéro à six, qui définit la nature et la fréquence des interventions pratiquées. Ce code va des espaces très soignés (zéro ou un), très artificialisés, et demandant une surveillance et une intervention constantes de la part de l'homme, comme les jardins publics situés dans le centre de la ville, à des espaces champêtres (cinq) où se développent la faune et la flore sauvages, comme dans des parcs périurbains ou des boisements. (La gestion différenciée ne s'applique guère au code six, qui désigne les terrains de culture ou les espaces ne comprenant que du mobilier urbain.)

La gestion différenciée offre une alternative à l'utilisation de produits phytosanitaires, mais n'en règle pas définitivement le problème. En effet, selon les normes esthétiques en vigueur, les espaces de code zéro ou un (entretien très soigné) impliquent l'utilisation de produits phytosanitaires susceptibles de se retrouver dans l'eau.

Pour un espace central de prestige, ou une jardinière, un entretien très poussé est pratiqué. Il n'est pas question de voir dépasser un liseron, une renoncule ou une pâquerette : ces plantes y sont indésirables. À l'inverse, dans des espaces plus naturels, comme les berges de la rivière ou bien des parcs périurbains, un traitement plus naturel est favorisé. On voit donc depuis plusieurs années apparaître des alternatives à des modèles très stricts et formels, même si ceux-ci restent encore la norme dans bien des villes françaises.

Source : Direction des Jardins (2001).

en l'amenant à plus de tolérance, par la mise en place d'actions de sensibilisation.

La question de la gestion alternative ou différenciée des espaces urbains a déjà été traitée et a fait l'objet de colloques et de publications (Allain, 1997). Cependant, cet aspect de l'entretien des villes a été essentiellement abordé à travers un questionnement technique. Peu de recherches, jusqu'à présent, ont été effectuées sur les aspects sociaux et culturels de ces nouvelles manières de jardiner (Agerri, 2004) et sur leur perception par les acteurs et les usagers de la ville, comme les agents des services d'entretien ou les habitants. Seule une étude a été réalisée sur l'acceptation de la gestion différenciée par les employés des services des jardins de la ville de Nantes (Emelianoff, 2005). De fait, il nous paraît pertinent d'intégrer dans un même questionnement la dimension technique de la gestion, les représentations dont la ville est l'objet et les relations qui s'élaborent entre les différents éléments humains et non humains qui composent l'espace urbain.

Nous sommes partis de deux hypothèses : que changer de techniques d'entretien n'est pas qu'un problème technique, mais ressort aussi de considérations sociales et culturelles; que l'objet «mauvaises herbes » s'élabore dans les interactions entre des modalités techniques d'entretien, des représentations des plantes et des espaces, des relations sociales. B. Latour (1994) définit les réseaux comme étant « réels comme la nature, narrés comme le discours, collectifs comme la société ». Dans la théorie de l'anthropologie symétrique, il préconise de s'interroger sur les réseaux existants entre des éléments qu'on a plutôt l'habitude de séparer dans les sociétés occidentales, comme l'approche naturaliste et l'approche politique des choses, les faits de nature d'un côté et les faits de culture de l'autre. La problématique s'organise autour du lien existant entre les acteurs sociaux, l'eau, les pesticides, les espaces, les modalités de leur entretien et la perception de la présence de «mauvaises herbes ». Nous présentons ici les résultats de l'étude sociologique qui a été un préalable à des actions de sensibilisation. Dans un premier point, nous tentons de cerner la définition des "mauvaises herbes » en relation avec ce qui définit la ville; puis, dans une seconde partie, nous les observons à partir des techniques d'entretien des espaces et des cultures professionnelles; enfin, dans une troisième section, nous nous interrogeons sur le rôle de ces plantes dans la vie sociopolitique de la ville.

La zone d'étude regroupe les quartiers de la Touche et Bourg l'Évêque (Fig. 1), deux quartiers à la périphérie du centre-ville. Ce choix est lié à la présence de la rivière de l'Ille qui les traverse. Le premier est constitué d'une zone d'habitat résidentiel et d'une zone d'habitat collectif qui représente environ trois quarts des logements $\mathrm{du}$ quartier, avec une population majoritairement composée d'employés et d'ouvriers. Le quartier de Bourg l'Évêque est essentiellement constitué d'habitat collectif, avec une surreprésentation des classes supérieures. Ces deux quartiers abritent une population vieillissante, mais connaissent un processus de rajeunissement. On y trouve des espaces et des jardins aux contours nets et bien délimités, des espaces «rustiques» comme les berges de l'Ille, des parterres de fleurs aux styles variés (parterres de pensées, parterres plus récents composés de plantes aromatiques, fleurs sur tiges...), mais aussi des espaces gérés d'une manière plus alternative, comme les pieds d'arbres ou certains parterres laissés enherbés. Les investigations ont essentiellement porté sur les espaces de circulation, et non sur les jardins et parcs proprement dits. 


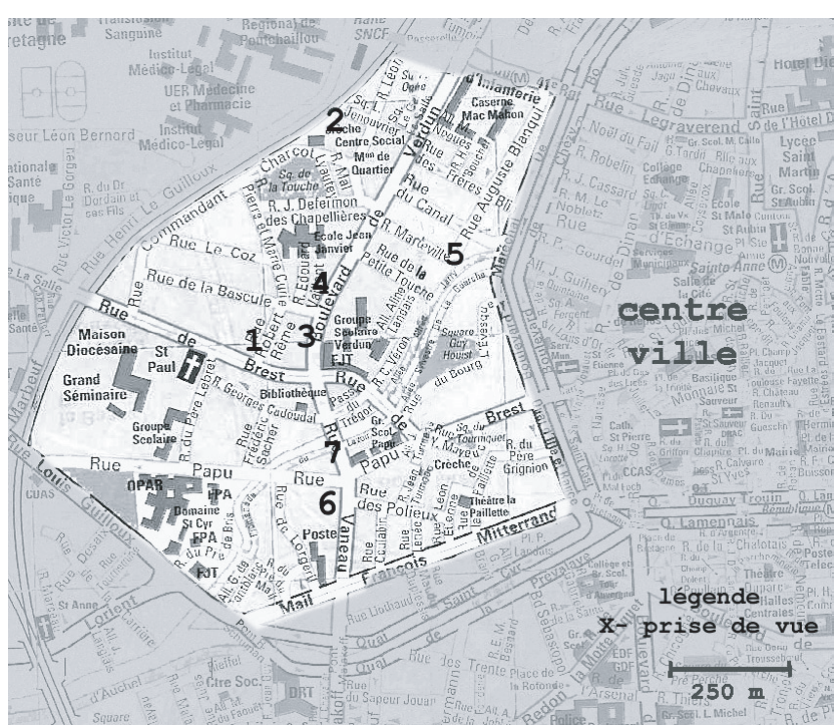

Fig. 1. Plan de la zone d'étude (fond de carte extrait du plan guide Blay).

Nous avons suivi, dans leurs déplacements, l'équipe des jardiniers de la ville et les employés de la propreté des rues qui travaillent dans ce secteur, interviewé des entrepreneurs privés de jardins, ainsi que des gestionnaires d'immeubles (syndics) et nous avons effectué des entretiens semi-directifs avec une vingtaine d'habitants $\mathrm{du}$ quartier. Lors de ces entretiens, nous les avons fait réagir sur des photographies de différents espaces du quartier : pâturin entre les pavés, adventices au pied d'une cabine téléphonique, haies bien taillées, végétation en bordure de rivière, etc. Pour compléter ces investigations, une randonnée dans le quartier avec un groupe d'une dizaine d'habitants a été organisée avec le comité de quartier de Bourg l'Évêque.

\section{Urbanité et catégories de végétation}

On peut distinguer dans la ville la structure matérielle, son dispositif écologique et la cité, espace politique ou religieux (Roncayolo, 1990). Le dispositif écologique - les éléments non humains - contribue autant que la cité à conférer à un espace son caractère de ville, son urbanité. On peut définir celle-ci comme un système particulier de relations sociales (Hannerz, 1983). Ce qui fait une ville, son urbanité, ne tient pas uniquement à la présence de populations humaines spécifiques, mais aussi à l'agencement des éléments non humains, dont la végétation. Celle-ci vient architecturer la ville et la dessiner (Peraldi, 1985 ; Gontier, 1993). Mais, pour cela, elle doit se conformer à certains critères : discipline des haies, des pelouses et des massifs de fleurs, régularité des compositions, tels que définis dans le modèle horticole (Allain, 1997), qui correspondent aux normes d'ordre et de propreté à partir desquelles sont définis les espaces urbanisés en Occident

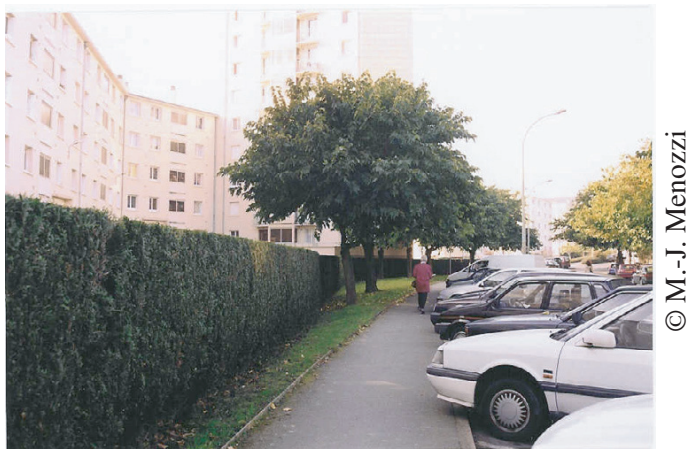

Photo 1. Paysage urbain propre et ordonné.

(Jeudy, 1991; Segaud, 1992). Ce modèle de nature policée, domestiquée (Photo 1), reste bien ancré dans le public ainsi que chez les aménageurs et les responsables urbains (Dubost, 1984 ; Lizet, 2003), et ce, malgré la diffusion d'autres modèles d'aménagement, comme une gestion différenciée, qui à Rennes coexiste avec une gestion horticole des espaces.

Bien que la ville soit une construction humaine, il y sourd toujours une part de nature et une ville n'est jamais totalement artificielle (Berque, 1993). À côté de cette « végétation institutionnelle et normée » (Blanc et al., 2005), coexiste une végétation spontanée, "naturelle». Dans la zone étudiée, entre quatre (au pied d'arbres) et une vingtaine d'espèces adventices (27 le long de la voie ferrée, 24 sur un îlot directionnel), selon la localisation, ont été identifiées en juillet 2001 (Diard et Aubin, 2001). Le nombre d'espèces est en augmentation par rapport à 2000, ce qu'on peut interpréter comme un effet de l'abandon du désherbant par les services de la ville sur cette zone pilote. Dans une perspective naturaliste, ces plantes spontanées participent à la biodiversité de la ville et sont des espèces caractéristiques du milieu urbain (Sukopp, 1997). Elles ont suscité l'intérêt de botanistes dès le début du XX $X^{\mathrm{e}}$ siècle, terrain d'investigations naturalistes encore peu reconnu à cette époque (Lizet, 1997). Cependant, cette perception des plantes urbaines spontanées comme caractéristique de la ville est peu partagée par les citadins.

Les investigations menées auprès des habitants nous ont permis de constater un manque d'observation et de reconnaissance des éléments végétaux du quartier. Beaucoup s'étonnaient que certaines des photos montrées fussent prises au bas de leur immeuble. Cela ne les a cependant pas empêché de produire des discours sur ce que doit être la végétation en ville. Les plantes spontanées sont peu connues, et seuls quelques noms ont été cités, comme les pissenlits, les orties, les ronces, les «épillets ", les myosotis, le chardon ou le plantain. Par ailleurs, ces plantes étaient généralement désignées sous le terme de "mauvaises herbes", et pas forcément perçues à leur place dans l'espace urbain - ce qui correspond précisément à la définition donnée par Harlan (1987). 


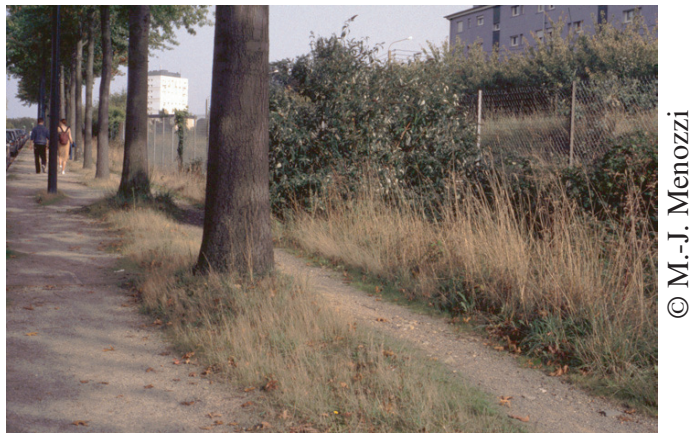

Photo 2. Des rats et des serpents ne sont-ils pas cachés derrière ces hautes herbes?

Les différentes catégories de végétation, horticole, spontanée, participent à la caractérisation des espaces et à leur délimitation. Les plantes spontanées renvoient à des espaces de "campagne ", «sauvages » ou « de nature ». La présence d'herbe au pied des arbres ou le long des trottoirs appelle des commentaires faisant référence à la campagne : "Ils attacheraient une chèvre au pied de l'arbre, ce serait tout nettoyé... » (coiffeur retraité). Dans les représentations des habitants, persiste l'image de la ville opposée à la campagne, telle qu'elle est pensée dans la tradition européenne (Ghorra-Gobin, 1997). Dans cette perspective, la présence de plantes adventices paraît inadaptée à la ville, dans la mesure où elles tendent à brouiller les frontières entre les différentes catégories d'espaces. Ces plantes sont, par contre, beaucoup plus tolérées dans les enclaves comme les berges de la rivière, ou le long de boulevards un peu périphériques, et appréciées à cet égard comme "bouts de campagne » ou comme éléments de nature permettant de contrebalancer la présence de la ville : « Je ne considère plus que c'est de la mauvaise herbe... parce que ça a un esprit... C'est la nature qui a voulu s'installer là... » (assistante sociale, 28 ans).

Les photos représentant des plantes adventices poussant le long de la voie ferrée inspirent des craintes liées à une nature perçue comme hostile, comme la peur des serpents, des rats, de la «vermine » qui grouilleraient dans les herbes hautes (Photo 2), notamment de la part de ceux qui habitent dans sa proximité immédiate. «Ça peut loger des animaux dangereux, des vipères, des rats [...]. C'est trop laisser-aller, trop haut, trop envahissant » (artiste, 36 ans). En revanche, pour les personnes résidant dans un autre endroit du quartier, cet espace apparaît «campagnard », voire naturel, et il est apprécié comme tel, du fait notamment des allées non goudronnées ; et, partant, la végétation adventice peut y trouver sa place. D'autres peurs inhérentes à la présence de plantes adventices sont aussi exprimées, comme des craintes liées à l'insécurité (le risque de masquer les panneaux de circulation) ou à des risques de glissades, ce qui renvoie à cette peur de la

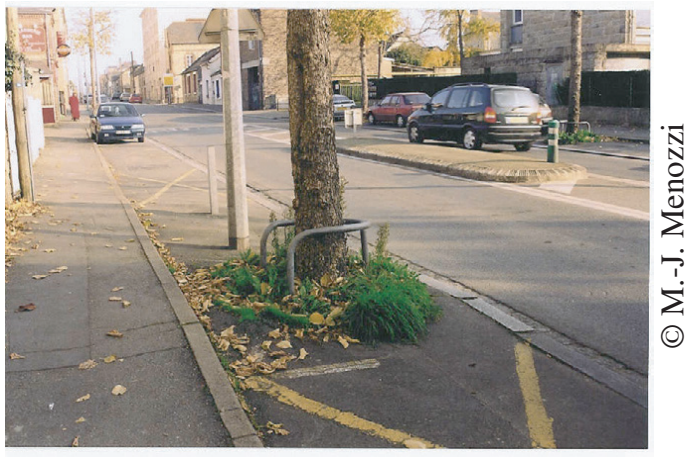

Photo 3. Adventices en pied d'arbre, des herbes pour les chèvres et les lapins?

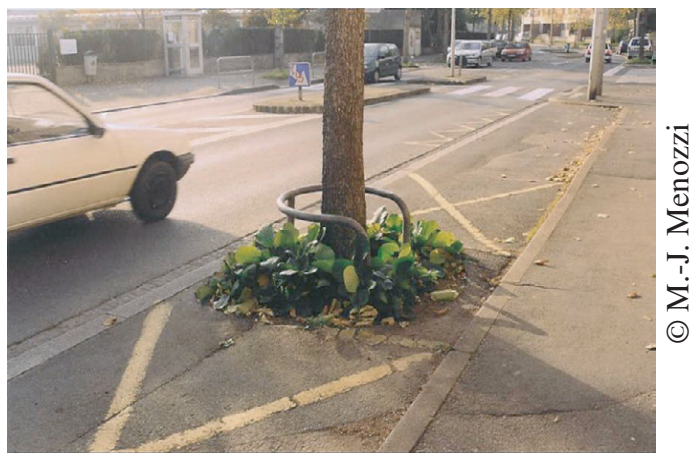

Photo 4. Quand les résidents préfèrent les plantes horticoles.

nature présente dans les sociétés occidentales dont nous parle Terrasson (1997).

La présence de ces plantes rend l'espace sale et négligé. La saleté est définie comme quelque chose de pas à sa place (Douglas, 1981). Elle partage cette définition avec les «mauvaises herbes", que les citadins assimilent à quelque chose de sale, qui nuit à la propreté de la ville. Beaucoup des photos présentant des plantes adventices appelaient des commentaires sur le manque de propreté de l'espace ${ }^{5}$. Le coiffeur en retraite fut rencontré en raison de l'attention qu'il portait à un pied d'arbre situé devant chez lui, dans le quartier enquêté. Il trouvait que les pieds d'arbres enherbés faisaient très négligés et, plutôt que de se plaindre auprès de la ville, il a décidé de mettre des plantes horticoles (des bergenias), afin d'empêcher les adventices de proliférer, ce qui répondait beaucoup plus à sa demande d'ordre et de propreté du milieu urbain (Photos 3 et 4). L'acceptation de la politique de la ville en matière de gestion alternative passe par la capacité à conserver des espaces propres : "L'initiative, je ne suis pas contre si ça reste propre quand même. Il faut que ce soit nettoyé, il ne faut pas laisser les herbes pousser trop haut, il faut enlever les mauvaises herbes » (commerçant, 40 ans).

\footnotetext{
${ }^{5}$ Lors d'une discussion informelle, un paysagiste a attiré notre attention sur le fait que des espaces «délaissés " peuplés de plantes adventices «attiraient » immanquablement détritus, papiers gras et autres déchets.
} 
Si on regarde les choses de manière plus attentive, on constate des distinctions dans la manière de percevoir les «mauvaises herbes » selon la catégorie socioprofessionnelle et selon l'âge. Dans les jardins des particuliers, les classes populaires et les personnes âgées sont les plus sensibles à l'ordre et à la propreté du jardin ainsi qu'à la présence de «mauvaises herbes » (Dubost, 1984). Il en est de même dans les espaces publics et collectifs. Les personnes âgées, notamment celles issues des classes populaires, portent un regard plus souvent négatif sur les plantes adventices. Leur présence est beaucoup plus tolérée par les personnes plus jeunes et les classes moyennes et supérieures. Elle est alors connotée à une idée de naturel qui peut contrebalancer le formalisme du «béton », idée d'autant plus marquée dans ces deux quartiers que l'habitat collectif y est très présent.

Les points de vue négatifs portés sur ces plantes les assimilent à quelque chose de sauvage, dans le sens de non maîtrisé par l'homme. Les points de vue positifs sont plus volontiers exprimés par l'utilisation du terme de «naturel », dans le sens d'opposé à artificiel, un «naturel » qui tendrait à répondre à une demande de nature en ville. Ces plantes peuvent alors être appréciées pour leur aspect esthétique, non pas les grandes tiges jaunes mais les plantes à fleurs comme les myosotis, les pâquerettes, qui sont l'objet d'une certaines tolérance parce que «c'est mignon » (assistante sociale). Si les plantes spontanées sont intéressantes pour les spécialistes, tels les écologues ou les biologistes, comme élément de biodiversité, ce terme n'évoque pas grand-chose pour les habitants et n'a guère de sens pour eux.

Ces plantes peuvent être tolérées à la condition que leur aspect sauvage soit soumis à une forme de socialisation par l'action de l'homme : «il faut que ce soit contrôlé, qu'on canalise un peu » (assistante sociale). Qu'il s'agisse des rues ou des berges de la rivière, dès que les plantes dépassent une certaine taille critique ( quand ça commence à faire $30 \mathrm{ou} 40 \mathrm{~cm}$ de haut, il faut faire quelque chose »: artiste, 36 ans) où dès qu'elles s'échappent des bordures délimitées, il surgit une crainte de l'envahissement par la végétation. De fait, les plantes basses, ou au ras du sol, sont plus acceptables dans l'environnement urbain que de grandes herbes. «L'herbe, ça ne me dérange pas, du moment qu'on la coupe. C'est de l'herbe trop sauvage qui dérange. Il ne faut pas la laisser déborder » (femme retraitée, classe supérieure). Pour les jardiniers de la ville, la difficulté réside dans le fait de laisser pousser les plantes tout en gardant le contrôle sur elles («il ne faut pas perdre le contrôle, c'est pas un espace naturel»). Tout semble alors être une question de bonne distance entre entretien et éléments sauvages présents en ville ${ }^{6}$.

\footnotetext{
${ }^{6}$ Clergeau et al. (1995) font le même constat à propos des oiseaux présents en ville. La nature sauvage «ne semble pouvoir exister en ville que propre, c'est-à-dire contrôlée, sans épine ni fiente ».
}

Pour produire une ville de qualité, il faut une nature, mais civilisée, contrôlée, travaillée, ordonnée (Calenge, 2003).

\section{Une question de techniques}

Sans l'invention du tuyau d'arrosage, de la tondeuse, ou bien des produits phytosanitaires, nos jardins et nos espaces verts n'auraient probablement pas le même aspect (Jenkins, 1994). La gestion horticole des villes est possible grâce aux progrès de la technologie et de l'industrie, comme l'apparition des engrais, des désherbants et des pesticides sur le marché (Allain, 1997). Pour nombre d'acteurs de l'entretien des espaces urbains que sont les employés des services des jardins, de la voirie, ou les entrepreneurs privés de jardins, la production de l'esthétique des espaces urbains, encore fortement imprégnée du modèle horticole et de la ville propre et ordonnée, ne peut se passer de l'utilisation de produits phytosanitaires.

Dans le modèle horticole, les herbes spontanées sont éliminées et, pour cela, la technique privilégiée est l'utilisation de produits désherbants, autant dans les espaces publics que privés. Les raisons généralement invoquées sont un moindre coût, ainsi qu'une rapidité d'exécution. Ce choix est aussi inscrit dans des modalités sociales et culturelles. Si les techniques et les gestes qui les composent peuvent être décrits indépendamment du contexte social et culturel, leur analyse ainsi que l'étude de leur développement ne peuvent se faire sans référence à ce contexte (Cresswell, 2002).

Participer au projet «zéro phyto » impliquait pour les services de la propreté de la ville ainsi que pour l'équipe de jardiniers d'abandonner totalement les produits phytosanitaires, ce qui nécessitait de trouver des méthodes alternatives à l'élimination des «mauvaises herbes ». Les équipes travaillant sur le quartier ont été partie prenante de l'expérience et ont relevé le défi. La présence d'un chef d'équipe imaginatif au niveau du service de la propreté a permis de chercher de nouvelles solutions. De même, l'équipe de jardiniers s'est lancée avec enthousiasme dans le projet et a quasiment abandonné l'usage de produits désherbants... quasiment dans la mesure où certains espaces ne pouvaient, selon eux, s'en passer.

Malgré cela, la réalisation de ce projet n'était pas dénuée de difficultés, car elle remettait en cause la culture professionnelle des employés des jardins et de la propreté. C. Emelianoff (2005) a bien décrit ce phénomène à propos de la mise en place de la gestion différenciée dans la ville de Nantes. Pour les jardiniers, en effet, de telles modalités de gestion vont à l'encontre de leur culture professionnelle et «l'espacement des tailles et des coupes, l'herbe haute et les pieds d'arbres non défrichés heurtent [leur] culture et [leur] sensibilité». Au moment de l'étude, 


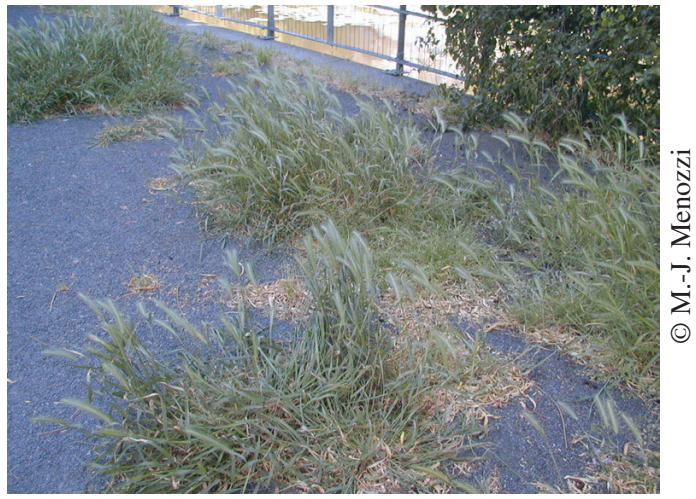

Photo 5. A-t-on l'impression d'avoir bien fait son travail si on laisse tout ça derrière soi?

certaines pratiques de gestion différenciée étaient appliquées depuis environ cinq ans dans le quartier : pieds d'arbres non désherbés, herbe non systématiquement enlevée le long des trottoirs... Pourtant, chez les jardiniers, existaient quelques "poches de résistance » à l'égard de «zéro phyto ». Par exemple, il n'était pas concevable de laisser des pâquerettes dans la pelouse ornée de massifs floraux (les pâquerettes atténuant le contraste entre fleurs et pelouse) et, au départ, le choix avait été fait de maintenir l'utilisation du désherbant sur cet espace.

Les services chargés de la propreté ont été confrontés à la difficulté de continuer à «faire de l'ordre et du propre» sans recourir aux produits désherbants. Dans le quartier pilote, les employés en ont abandonné l'usage, non sans peine. Comme alternative, ils ont trouvé la raclette à neige, qui s'est avérée être, selon eux, l'outil le plus adéquat pour ôter les plantes adventices présentes le long des trottoirs et des caniveaux (Photo 6). Mais n'utiliser aucun désherbant impliquait aussi de laisser des herbes derrière leur passage, ce qui allait à l'encontre de leur conception aussi bien de l'espace urbain que de la qualité du travail effectué. Comment avoir l'impression $\mathrm{d}^{\prime}$ avoir bien fait son travail, si on laisse derrière soi des «mauvaises herbes » (Photo 5)?

L'utilisation de produits pesticides est tellement intégrée dans les pratiques que leur arrêt est loin d'aller de soi. Ils sont réputés être efficaces, pas chers, rapides et faciles d'emploi. Leur usage est totalement banalisé par nombre d'acteurs sociaux, et ils semblent être là pour régler rapidement tous les problèmes posés par l'horticulture et le jardinage. Exclure les pesticides tout en conservant les mêmes aspects aux espaces induit une dépense de temps et un coût supplémentaire, selon les jardiniers de la ville et les entrepreneurs privés. Et, pour la plupart d'entre eux, il est difficile de ne plus du tout en utiliser : «[...] il en faut quand même un peu » est une réponse plusieurs fois entendue. Pour certains professionnels, employer des produits pesticides est l'une des seules réponses qu'on peut apporter aux exigences de la société contemporaine, à savoir faire vite pour pas cher : «La nature, faut qu'on la maîtrise un peu. Avant, on prenait le temps de le faire. Maintenant, tout est question de temps et de rentabilité » (entrepreneur privé de jardins).

A contrario, il est intéressant de constater les perceptions négatives dont le désherbeur thermique ${ }^{7}, 1^{\prime}$ une des techniques alternatives aux produits chimiques, peut être l'objet. Il s'agit d'une sorte de petit tracteur sur lequel est installée une bouteille de gaz. Il produit une flamme $q^{\prime}$ 'on dirige sur les plantes indésirables, qui sont ainsi brûlées. Cette machine se déplace à une vitesse inférieure à $2 \mathrm{~km} / \mathrm{h}$, ce qui entre en contradiction avec une certaine idée de la vitesse, inhérente à la vie urbaine. Cela a amené certains résidents du quartier à dire que les jardiniers «étaient payés par la ville à ne rien faire »...

L'« évidence » de l'utilisation des désherbants chimiques s'inscrit aussi dans les représentations dont ils sont l'objet. Ces produits sont considérés par nombre d'acteurs comme étant à la pointe du progrès et sans équivalent au niveau de l'efficacité. Ne plus utiliser de pesticides, ce serait « retourner cinquante ans en arrière » (entrepreneur de jardins), vers des temps archaïques. On peut aussi s'interroger sur le "surinvestissement magique » dont ils sont l'objet, ainsi que l'observe LelièpvreBotton (1997, p. 40) à propos du développement des techniques: "Dans nos sociétés, la diffusion des acquis technologiques (parce qu'elle ne s'est pas accompagnée d'une vulgarisation sérieuse et efficace des connaissances impliquées par ces procédés) a eu pour effet ultime une naïveté généralisée quant aux possibilités techniques; cela explique le réinvestissement magique : les modernes croient en la "toute-puissance" de la technique. »

Cependant, les jardiniers, notamment, ont élaboré d'autres modes de valorisation de leur travail. Modifier les techniques d'entretien impliquait pour eux un changement de relations avec les plantes : non plus un traitement de masse par le désherbant, mais une gestion sélective des plantes, certaines étant gardées, d'autres, enlevées. Cela nécessitait de leur part une observation plus attentive des plantes et a suscité un intérêt accru pour leur identification (un herbier a même été réalisé), ainsi que pour la vie animale et végétale qu'elles favorisent. Cela contribue aussi à brouiller les frontières entre les catégories de plantes horticoles et sauvages. Les plantes horticoles reçoivent un entretien plus libre: on les laisse dépasser des bordures pour cacher des «mauvaises

\footnotetext{
7 Tout le monde n'est pas d'accord au sein du service des jardins, dans le cadre d'une gestion «durable», pour utiliser le désherbeur thermique dans la mesure où il est consommateur d'énergie. Pour le directeur, il conduit à s'interroger sur les bonnes questions à se poser à l'égard des «mauvaises herbes » : non pas se demander comment désherber (question essentiellement technique), mais pourquoi désherber (question d'ordre socio-technique, impliquant de prendre en compte les modalités de représentation des espaces et de leur gestion).
} 


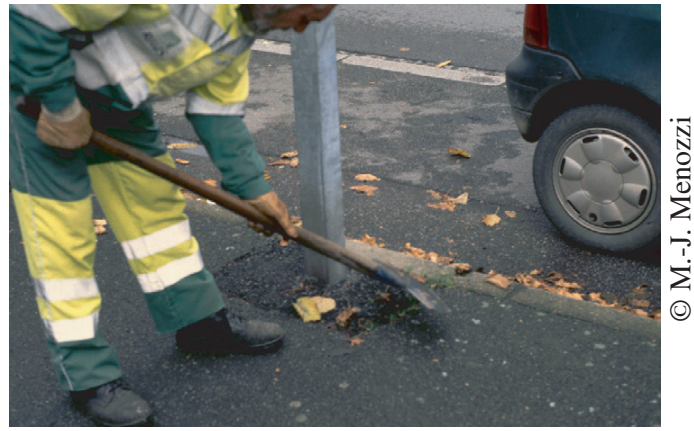

Photo 6. La raclette à neige, une alternative aux herbicides.

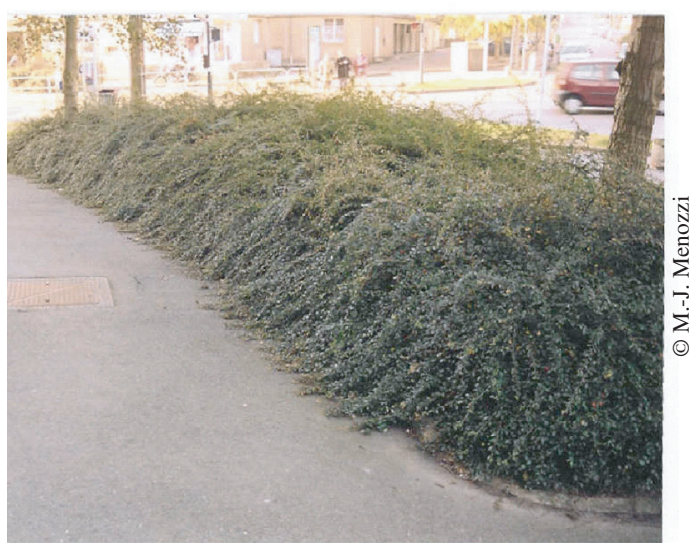

Photo 7. Bordure horticole « libre».

herbes », ou bien on les taille moins pour gagner du temps et compenser ainsi le temps supplémentaire mis a désherber (Photo 7). Quant aux plantes spontanées, elles sont intégrées à l'espace urbain par la gestion qui leur est appliquée : le pâturin est tondu comme un gazon, de même que les pieds d'arbres, les grandes herbes sont fauchées.

\section{La «mauvaise herbe ", une manifestation du politique?}

Constitutives de la ville au même titre que les humains, les plantes urbaines participent aussi aux modalités d'organisation sociopolitiques de l'espace. Cela peut se manifester de diverses manières. Dans nombre de société, les arbres plantés sont un symbole du pouvoir en place. La pelouse américaine prend une forte traduction politique et, sous l'unification de l'espace grâce aux vertes pelouses, est exprimé l'idéal démocratique américain, celui d'un espace géographique, mais aussi sociologique, unifié ${ }^{8}$.

\footnotetext{
${ }^{8}$ Une exposition a été organisée en 1998 à Québec par le Centre Canadien d'architecture (CCA) sur la pelouse américaine, où était notamment souligné le lien entre le développement de la démocratie américaine et le développement des espaces de pelouse. Voir le site Internet : http://www.cca.qc.ca/ pages/Niveau3.asp? page $=$ depliant\&lang $=$ fra
}

De même, les «mauvaises herbes » participent de la vie sociale et politique urbaine. Une des difficultés à modifier les modalités d'entretien des espaces réside pour partie dans le fait que les plantes présentes en ville, les adventices comme les horticoles, font partie des relations entretenues entre les différentes catégories d'acteurs en milieu urbain. En tout cas, leur gestion s'insère dans la gestion globale de la ville et dans la manière dont la municipalité administre ses citoyens. Cela illustre le constat émis par B. Latour (1993), à savoir que les relations sociales ne sont pas faites que de relations sociales, mais sont aussi constituées des objets qui peuplent le social.

L'entretien de la ville participe à l'image de celle-ci et à son identité et témoigne pour partie de l'intérêt que les élus manifestent envers les citoyens. Pour les habitants, l'entretien des espaces est important dans la mesure où « c'est représentatif du souci municipal sur le bien-être citoyen » (assistante sociale). À cet égard, il est intéressant d'observer quelle interprétation politique les habitants de certains quartiers font de la gestion différenciée. Si cette méthode a essentiellement été présentée à travers ses caractéristiques techniques et écologiques, la dimension sociale en a été occultée dans les campagnes de communication. Le principe de la gestion différenciée établit une différenciation des espaces selon un gradient d'entretien, allant de très entretenu à espace champêtre, gradient qui s'organise du centre vers la périphérie. Les espaces centraux de la ville, considérés comme des espaces de prestige, sont soumis à une pression d'entretien élevée (comme, par exemple, le jardin du Thabor à Rennes), au contraire d'espaces plus périphériques, qui vont être gérés d'une manière plus libre. Cette organisation technique de l'entretien porte en elle une représentation de la ville organisée en cercles concentriques, répartition de l'entretien qui est mal perçue par les habitants des quartiers périphériques, qui en donnent volontiers une lecture politique. Ce procédé est mal accepté par les commerçants du quartier de la Touche, qui se sentent délaissés au profit de ceux du centre-ville. L'un d'entre eux se plaignait des "épillets » présents au pied des arbres : «C'est pas dans le centre-ville qu'on verrait ça. » Lors des investigations effectuées, le maire en personne a été pris à partie, d'aucuns se demandant s'il n'oubliait pas certains de ses administrés. Mettre en œuvre une politique d'entretien selon un gradient de pression décroissant du centre vers la périphérie est interprété par les habitants de la périphérie comme un désintérêt envers eux de la part de la municipalité. Selon eux, on s'occupe des habitants du centre de la ville, et ceux de la périphérie, on les délaisse : pour preuve, on n'enlève plus les «mauvaises herbes ». «Ho! Monsieur le maire, vous nous oubliez? » demandait une femme interrogée (sans profession, 48 ans). «Hou! Hou! Monsieur le maire, allez, enlevez-moi tout ça, nettoyez-moi ça » (homme sans profession, 34 ans). Interprété d'un point de vue social, 
ce principe de gestion organise une différenciation des types d'espaces verts, mais aussi, selon la lecture qui en est faite par les habitants, des catégories de population présentes en ville. Ainsi, la distinction centre/périphérie est notamment ressentie parmi les classes populaires qui habitent dans la périphérie. Aux habitants du centre et aux quartiers prestigieux, un entretien soigné ; aux habitants des quartiers périphériques et péricentraux, les «mauvaises herbes » dans les pelouses et le long des trottoirs. Ils perçoivent la présence de «mauvaises herbes » comme étant de la saleté et, partant, un défaut de gestion de la ville... et de prise en considération de ses habitants. Cela illustre bien le propos de M. Segaud (1992, p. 12) : «Le propre est conçu désormais comme un élément primordial de l'urbanité et du même coup, de la civilité. On peut même parler d'enjeu emblématique : celui de l'image du quartier ou de la ville, qui - on le sait bien maintenant - participe à part entière des politiques locales : une ville propre, n'est-ce pas une ville ordonnée, gérée, contrôlée? »L'un des arguments avancés est le fait que tous les habitants "paient des impôts locaux", donnant droit à un minimum de considération et d'enlèvement des «mauvaises herbes ». Les employés de la propreté eux-mêmes s'interrogeaient sur ces manières de faire : «Il y a deux, trois couronnes, du plus au moins nickel... alors que tout le monde paie des impôts ! Pourquoi à la périphérie ils n'ont pas droit à quelque chose de nickel ? Tout le monde n'est pas au même diapason !... Trois couronnes, pour moi, c'est pas normal ${ }^{9}$. »

Toutefois, c'est moins la présence de plantes spontanées qui importe que la perception qu'en ont les habitants, laquelle est fortement liée à l'impression d'entretien ou non. Le même espace peuplé de plantes adventices sera perçu différemment si les riverains ont l'impression que c'est entretenu par les jardiniers ou non. D'ailleurs, ceux-ci nous expliquaient que, dans certains endroits, ils passaient sans rien faire, mais que cette présence rassurait les habitants qui les voyaient passer... et qui parfois les félicitaient pour le bon entretien de l'espace, alors que celui-ci était laissé à un entretien spontané! Si ces plantes sont intégrées dans un système de relations avec les acteurs de la ville, comme les jardiniers par exemple, un autre regard sera porté sur elles. De même, si les jardiniers expliquent pourquoi ils laissent ces plantes, leur acceptation par les riverains en sera accrue.

\section{En conclusion, et la qualité de l'eau ?}

Si la préoccupation initiale était la qualité de l'eau, nous n'avons guère abordé celle-ci jusqu'à présent. En effet, il est apparu que, pour les personnes enquêtées, il était peu évident de faire un lien entre la qualité de

\footnotetext{
${ }^{9}$ Ce jardinier se réfère à l'espace urbain organisé en espace central, espace péricentral et espace périphérique.
}

l'eau et l'aspect pris par les espaces. Elles n'établissaient pas de relation entre leur propre pratique, leur demande d'espaces «propres », et la qualité de l'eau, ou bien elles l'admettaient sur le mode fataliste « on est tous des pollueurs ». Peu de choses, sinon rien, permettent à ces acteurs de se sentir concernés de manière concrète par ce fait, et leur surprise était grande quand on leur annonçait l'objet de l'enquête. Cela n'est pas spécifique à la question de la demande de gestion des espaces urbains; des constats similaires sont faits à propos de la pollution urbaine, peu reliée à sa propre pratique de citadin (Blanc et al., 2005). Si, dans l'absolu, la plupart des personnes interrogées se disent concernées par les problèmes d'environnement, pour la plupart d'entre elles, la résolution de ces problèmes n'est pas de leur ressort, et la responsabilité de la pollution est souvent rejetée sur les autres catégories d'acteurs. Les pratiques agricoles, notamment, sont incriminées. La mise en place de telles actions ne doit pas nuire à la lisibilité de la ville en tant que ville et la présence de plantes adventices est d'autant plus acceptable qu'elle est intégrée à l'urbanité de la ville, à ses interactions, en étant soumise à un processus de socialisation par les jardiniers. Malgré la demande de nature en ville, on voit que continue de dominer l'idée de la ville comme lieu d'artifice, où prédomine «la main de l'homme », où la nature n'a sa place que dans des espaces définis et soumise à une forme d'entretien et de contrôle de la part de l'homme.

Nous avons pu lier dans un même réseau des faits techniques, écologiques, politiques, sociaux, culturels, ainsi que des représentations. Pourtant, pour nombre d'acteurs sociaux, ces différents aspects d'une même réalité continuent d'être séparés. Pour reprendre les expressions de B. Latour (1994), nous vivons ce tissu sans couture comme étant au contraire couturé. La «mauvaise herbe » et l'aspect des espaces sont des thèmes qui mobilisent des savoirs et des compétences techniques. Par ailleurs, ces herbes, présentes ou absentes, s'inscrivent dans des réseaux sociaux de relation et participent à l'organisation de la ville et aux relations qui en définissent l'urbanité. On se rend compte qu'il n'y a guère de liens établis entre ces deux aspects des choses ${ }^{10}$. En mettant au jour ces réseaux, l'analyse sociologique peut être un préalable à la mise en place d'actions opérationnelles de préservation de la qualité de l'eau et de gestion favorisant la biodiversité, dans la mesure où l'efficacité des actions engagées implique qu'une reconnaissance de ces réseaux existants soit effectuée, et qu'une mise en relation des éléments soit réalisée.

\footnotetext{
${ }^{10}$ Cependant, des responsables de services d'espaces verts de différentes villes, rencontrés par la suite, disent de plus en plus être conscients que la mise en place d'une gestion alternative des espaces urbains relève plus de difficultés d'ordre social et culturel que de difficultés techniques proprement dites.
} 


\section{Références}

Agerri, G., 2004. La Nature sauvage et champêtre dans les villes : origine et construction de la gestion différenciée des espaces verts publics et urbains. Le cas de la ville de Montpellier. Thèse de doctorat, Engref, Paris.

Allain, Y.M, 1997. La ville : un territoire nouveau pour la nature? La gestion différenciée en Europe, in Lizet, B., Wolf, A.E, Celecia, J. (Eds), Sauvages dans la ville, Jatba, XXXIX, 2, 199217.

Berque, A., 1993. Du geste à la cité : formes urbaines et lien social au Japon, Paris, Gallimard.

Blanc, N., Bridier, S., Glatron, S., Grésillon, L., Cohen, M., 2005. Appréhender la ville comme (mi)lieu de vie. L'apport d'un dispositif interdisciplinaire de recherche, in Mathieu, N., Guermond, Y. (Eds), La Ville durable, du politique au scientifique, Paris, Cemagref/Cirad/Ifremer/Inra Éditions, 261-281.

Callenge, C., 2003. Idéologie verte et rhétorique paysagère, Communications, 74, 33-45.

Clergeau, P., Sauvage, A., Lemoine, A., Marchand, J.-P., Dubs, F., Mennechez, G., 1995. Quels oiseaux dans la ville ? Une étude pluridisciplinaire d'un même gradient urbain, Les Annales de la recherche urbaine, 74, 119-130.

Cresswell, R., 2002. Geste technique, fait social total. Le technique est-il dans le social ou face à lui ?, Techniques et cultures, 40, 125-151.

Diard, L., Aubin, 2001. Relevé floristique : dynamique de la végétation. Rapport, Ville de Rennes, Direction des jardins.

Direction des Jardins, 2001. Code qualité, Ville de Rennes.

Douglas, M., 1981. De la souillure, Paris, Maspéro.

Dubost, F., 1984. Côté jardins, Paris, Scarabées et Compagnie.

Dubost, F., Lizet, B., 2003. La nature dans la cité. De l'hygiénisme au développement durable, Communications, 74, 5-17.

Emelianoff, C., 2005. La ville durable en quête de transversalité, in Mathieu, N., Guermond, Y. (Eds), La Ville durable, du politique au scientifique. Paris, Cemagref/Cirad/Ifremer/Inra Éditions, 129-142.

Ghorra-Gobin, C., 1997. La ville américaine. De l'idéal pastoral à l'artificialisation de l'espace naturel, Les Annales de la recherche urbaine, 74, 69-74.

Reçu le 21 octobre 2005. Accepté le 7 décembre 2006.
Gontier, C., 1993. L'Arbre d'ornement, marqueur symbolique et social des espaces publics urbains : le cas des politiques d'urbanisation de la zone Fos-Étang de Berre. Rapport, SRETIE/CERFISE, Paris/Marseille.

Hannerz, U., 1983. Explorer la ville, Paris, Éditions de Minuit.

Harlan, J.R, 1987. Les Plantes cultivées et l'homme, Paris, PUF.

Idea Recherche, Ceresa, 2002. Adaptation de l'entretien des espaces publics aux exigences de qualité des eaux : diagnostic social et mise en place d'une démarche participative, Ville de Rennes.

Jenkins, V. Scott, 1994. The Lawn: A History of an American Obsession, Washington/London, Smithsonian Institution Press.

Jeudy, H.P, 1991. Le choix public du propre. Une propriété des sociétés modernes, Les Annales de la Recherche urbaine 53, 102-107.

Latour, B., 1993. Petite leçon de sociologie des sciences, Paris, La Découverte.

Latour, B., 1994. Nous n'avons jamais été modernes : essai d'anthropologie symétrique, Paris, La Découverte.

Lelièpvre-Botton, S., 1997. L'Essor technologique et l'idée du progrès, Paris, Ellipses.

Lizet, B., 1997. Prologue, in Lizet, B., Wolf, A.E, Celecia, J. (Eds), Sauvages dans la ville, Jatba, XXXIX, 2, 9-13.

Observatoire de l'eau, 2004. Les Cahiers 2004, diff. Rennes Métropoles.

Peraldi, M., 1985. Les espaces verts et leurs mythes, Le Genre humain, 12, 203-216.

Roncayolo, M., 1990. La Ville et ses territoires, Paris, Gallimard.

Segaud, M., 1992. Introduction, in Segaud, M. (Ed.), Le Propre de la ville: pratiques et symboles, La Garenne-Colombes, Éditions de l'Espace européen.

Sukopp, 1997. History of the flora and vegetation of Berlin and their conservation. La gestion différenciée en Europe, in Lizet, B., Wolf, A.E, Celecia, J. (Eds), Sauvages dans la ville, Jatba, XXXIX, 2, 265-283.

Terrasson, F., 1997. La Peur de la nature, Paris, Sang de la terre. 